\title{
Applications of Group Analysis to the Three-Dimensional Equations of Fluids with Internal Inertia*
}

\author{
Piyanuch SIRIWAT and Sergey V. MELESHKO
}

School of Mathematics, Suranaree University of Technology, Nakhon Ratchasima, 30000, Thailand

E-mail: fonluang@yahoo.com,sergey@math.sut.ac.th

Received October 31, 2007, in final form February 12, 2008; Published online February 24, 2008

Original article is available at http://www.emis.de/journals/SIGMA/2008/027/

\begin{abstract}
Group classification of the three-dimensional equations describing flows of fluids with internal inertia, where the potential function $W=W(\rho, \dot{\rho})$, is presented. The given equations include such models as the non-linear one-velocity model of a bubbly fluid with incompressible liquid phase at small volume concentration of gas bubbles, and the dispersive shallow water model. These models are obtained for special types of the function $W(\rho, \dot{\rho})$. Group classification separates out the function $W(\rho, \dot{\rho})$ at 15 different cases. Another part of the manuscript is devoted to one class of partially invariant solutions. This solution is constructed on the base of all rotations. In the gas dynamics such class of solutions is called the Ovsyannikov vortex. Group classification of the system of equations for invariant functions is obtained. Complete analysis of invariant solutions for the special type of a potential function is given.
\end{abstract}

Key words: equivalence Lie group; admitted Lie group; optimal system of subalgebras; invariant and partially invariant solutions

2000 Mathematics Subject Classification: 76M60; 35Q35

\section{Introduction}

The article focuses on group classification of a class of dispersive models $[1]^{1}$

$$
\begin{aligned}
& \dot{\rho}+\rho \operatorname{div}(u)=0, \quad \rho \dot{u}+\nabla p=0, \\
& p=\rho \frac{\delta W}{\delta \rho}-W=\rho\left(\frac{\partial W}{\partial \rho}-\frac{\partial}{\partial t}\left(\frac{\partial W}{\partial \dot{\rho}}\right)-\operatorname{div}\left(\frac{\partial W}{\partial \dot{\rho}} u\right)\right)-W,
\end{aligned}
$$

where $t$ is time, $\nabla$ is the gradient operator with respect to the space variables, $\rho$ is the fluid density, $u$ is the velocity field, $W(\rho, \dot{\rho})$ is a given potential, "dot" denotes the material time derivative: $\dot{f}=\frac{d f}{d t}=f_{t}+u \nabla f$, and $\frac{\delta W}{\delta \rho}$ denotes the variational derivative of $W$ with respect to $\rho$ at a fixed value of $u$. These models include the non-linear one-velocity model of a bubbly fluid (with incompressible liquid phase) at small volume concentration of gas bubbles (Iordanski [2], Kogarko [3], Wijngaarden [4]), and the dispersive shallow water model (Green \& Naghdi [5], Salmon [6]). For the Green-Naghdi model, the potential function is [1]

$$
W(\rho, \dot{\rho})=\rho\left(3 g \rho-\varepsilon^{2} \dot{\rho}^{2}\right) / 6,
$$

${ }^{\star}$ This paper is a contribution to the Proceedings of the Seventh International Conference "Symmetry in Nonlinear Mathematical Physics" (June 24-30, 2007, Kyiv, Ukraine). The full collection is available at http://www.emis.de/journals/SIGMA/symmetry2007.html

${ }^{1}$ See also references therein. 
where $g$ is the gravity, $\varepsilon$ is the ratio of the vertical length scale to the horizontal length scale. For the Iordanski-Kogarko-Wijngaarden model, the potential function is [1]

$$
W(\rho, \dot{\rho})=\rho\left(c_{2} \rho_{20} \varepsilon_{20}\left(\rho_{20}\right)-2 \pi n \rho_{10} R^{3} \dot{R}^{2}\right),
$$

where

$$
\frac{4}{3} \pi n R^{3}=\left(\frac{1}{\rho}-\frac{c_{1}}{\rho_{10}}\right), \quad \rho_{20}=c_{2}\left(\frac{1}{\rho}-\frac{c_{1}}{\rho_{10}}\right)^{-1},
$$

$\varepsilon_{20}$ is the internal energy of the gas phase, $c_{1}$ and $c_{2}$ are the mass concentrations of the liquid and gas phases, $n$ is the number of bubbles per unit mass, $\rho_{10}$ and $\rho_{20}$ are the physical densities of components. The quantities $c_{1}, c_{2}, n$ and $\rho_{10}$ are assumed constant.

One of the methods for studying of differential equations is group analysis [7]. Many applications of group analysis to partial differential equations are collected in [8]. Group analysis beside construction of exact solutions provides a regular procedure for mathematical modeling by classifying differential equations with respect to arbitrary elements. An application of group analysis involves several steps. The first step is the group classification with respect to arbitrary elements. This paper considers group classification of equations (1) in the three-dimensional case, where the function $W_{\dot{\rho} \dot{\rho}}$ satisfies the condition $W_{\dot{\rho} \dot{\rho}} \neq 0$. Notice that for $W_{\dot{\rho} \dot{\rho}}=0$ or $W(\rho, \dot{\rho})=\dot{\rho} \varphi(\rho)+\psi(\rho)$, the momentum equation becomes

$$
\dot{u}+\psi^{\prime \prime} \rho_{x}=0 \text {. }
$$

Hence in the case $W_{\dot{\rho} \dot{\rho}}=0$, equations (1) are similar to the gas dynamics equations. This case has been completely studied [9] (see also [10]).

The one-dimensional case of equations (1) was studied in [11]. As in the case of the gas dynamics equations there are differences in the group classifications of one-dimensional and three-dimensional equations.

Another part of this paper is devoted to a special vortex solution. This solution was introduced by L.V. Ovsyannikov [12] for ideal compressible and incompressible fluids. This is a partially invariant solution, generated by the Lie group of all rotations. L.V. Ovsyannikov called it a "singular vortex". It is related with a special choice of non-invariant function. He also gave complete analysis of the overdetermined system corresponding to this type of partially invariant solutions: all invariant functions satisfy the well-defined system of partial differential equations with two independent variables. The main features of the fluid flow, governed by the obtained solution, were pointed out in [12]. It was shown that trajectories of particles are flat curves in three-dimensional space. The position and orientation of the plane, which contains the trajectory, depends on the particle's initial location. Later particular solutions of the system of partial differential equations for invariant functions were studied in $[13,14,15,16]$. For some other models, this type of partially invariant solutions was considered in [17, 18]. Exact solutions in fluid dynamics generated by a rotation group are of great interest by virtue of their high symmetry. The classical spherically symmetric solutions is one of the particular cases of such solutions.

In this manuscript a singular vortex of the mathematical model of fluids with internal inertia is studied. Complete group classification of the system of equations for invariant functions is given. All invariant solutions for this system are presented.

\section{Equivalence Lie group}

Since the function $W$ depends on the derivatives of the dependent variables, for the sake of simplicity of finding the equivalence Lie group, new dependent variables are introduced:

$$
u_{5}=\dot{\rho}, \quad \phi_{1}=W, \quad \phi_{2}=W_{\rho}, \quad \phi_{3}=W_{\dot{\rho}},
$$


where $u_{4}=\rho$ and $x_{4}=t$. An infinitesimal operator $X^{e}$ of the equivalence Lie group is sought for in the form [19]:

$$
X^{e}=\xi^{i} \partial_{x_{i}}+\zeta^{u_{j}} \partial_{u_{j}}+\zeta^{\phi_{k}} \partial_{\phi_{k}},
$$

where all coefficients $\xi^{i}, \zeta^{u_{j}}$ and $\zeta^{\phi_{k}}(i=1,2, j=1,2,3,4,5, k=1,2,3)$ are functions of the variables $^{2} x_{i}, u_{j}$ and $\phi_{k}$. Hereafter a sum over repeated indices is implied.

The coefficients of the prolonged operator are obtained by using the prolongation formulae:

$$
\begin{aligned}
& \zeta^{u_{\alpha, i}}=D_{i}^{e} \zeta^{u_{\alpha}}-u_{\alpha, j} D_{i}^{e} \xi^{x_{j}} \quad(i=1,2,3,4), \\
& D_{i}^{e}=\partial_{x_{i}}+u_{\alpha, i} \partial_{u_{\alpha}}+\left(\rho_{x_{i}} W_{\beta, 1}+\dot{\rho}_{x_{i}} W_{\beta, 2}\right) \partial_{W_{\beta}},
\end{aligned}
$$

where $\alpha=\left(\alpha_{1}, \alpha_{2}, \alpha_{3}, \alpha_{4}\right)$ and $\beta=\left(\beta_{1}, \beta_{2}\right)$ are multiindices $\left(\alpha_{i} \geq 0, \beta_{i} \geq 0\right)$,

$$
\begin{gathered}
\left(\alpha_{1}, \alpha_{2}, \alpha_{3}, \alpha_{4}\right), \quad j=\left(\alpha_{1}+\delta_{1 j}, \alpha_{2}+\delta_{2 j}, \alpha_{3}+\delta_{3 j}, \alpha_{4}+\delta_{4 j}\right), \\
u_{\left(\alpha_{1}, \alpha_{2}, \alpha_{3}, \alpha_{4}\right)}=\frac{\partial^{\alpha_{1}+\alpha_{2}+\alpha_{3}+\alpha_{4}} u}{\partial x_{1}^{\alpha_{1}} \partial x_{2}^{\alpha_{2}} \partial x_{3}^{\alpha_{3}} \partial t^{\alpha_{4}}}, \quad W_{\left(\beta_{1}, \beta_{2}\right)}=\frac{\partial^{\beta_{1}+\beta_{2}} W}{\partial \rho^{\beta_{1}} \partial \dot{\rho}^{\beta_{2}}} .
\end{gathered}
$$

The conditions that $W$ does not depend on $t, x_{i}, u_{i}(i=1,2,3)$ give that

$$
\zeta_{x_{i}}^{u_{k}}=0, \quad \zeta_{u_{j}}^{u_{k}}=0, \quad \zeta_{x_{i}}^{W}=0, \quad \zeta_{u_{j}}^{W}=0 \quad(i=1,2,3,4, j=1,2,3, k=4,5) .
$$

With these relations the prolongation formulae for the coefficients $\zeta^{W_{\beta}}$ become:

$$
\zeta^{W_{\beta, i}}=\widetilde{D}_{i}^{e} \zeta^{W_{\beta}}-W_{\beta, 1} \widetilde{D}_{i}^{e} \zeta^{u_{4}}-W_{\beta, 2} \widetilde{D}_{i}^{e} \zeta^{u_{5}} \quad(i=1,2),
$$

where

$$
\widetilde{D}_{1}^{e}=\partial_{\rho}+W_{\beta, 1} \partial_{W_{\beta}}, \quad \widetilde{D}_{2}^{e}=\partial_{\dot{\rho}}+W_{\beta, 2} \partial_{W_{\beta}} .
$$

For constructing the determining equations and solving them, the symbolic computer program Reduce [20] was applied. Calculations yield the following basis of generators of the equivalence Lie group

$$
\begin{aligned}
& X_{1}^{e}=\partial_{x_{1}}, \quad X_{2}^{e}=\partial_{x_{2}}, \quad X_{3}^{e}=\partial_{x_{3}}, \quad X_{4}^{e}=t \partial_{x_{1}}+\partial_{u_{1}}, \quad X_{5}^{e}=t \partial_{x_{2}}+\partial_{u_{2}}, \\
& X_{6}^{e}=t \partial_{x_{3}}+\partial_{u_{3}}, \quad X_{7}^{e}=u_{2} \partial_{u_{2}}-u_{1} \partial_{u_{2}}+x_{2} \partial_{x_{1}}-x_{1} \partial_{x_{2}}, \\
& X_{8}^{e}=u_{3} \partial_{u_{1}}-u_{1} \partial_{u_{3}}+x_{3} \partial_{x_{1}}-x_{1} \partial_{x_{3}}, \quad X_{9}^{e}=u_{3} \partial_{u_{2}}-u_{2} \partial_{u_{3}}+x_{3} \partial_{x_{2}}-x_{2} \partial_{x_{3}}, \\
& X_{10}^{e}=\partial_{t}, \quad X_{11}^{e}=t \partial_{t}+x_{i} \partial_{x_{i}}, \quad X_{12}^{e}=\partial_{W}, \quad X_{13}^{e}=\rho \partial_{W}, \quad X_{14}^{e}=\rho \partial_{W}, \\
& X_{15}^{e}=\dot{\rho} \partial_{\dot{\rho}}+\rho \partial_{\rho}+W \partial_{W}, \quad X_{16}^{e}=x_{i} \partial_{x_{i}}+u_{i} \partial_{u_{i}}-2 \rho \partial_{\rho} .
\end{aligned}
$$

Here, only the essential part of the operators $X_{i}^{e}$ is written. For example, the operator $X_{11}^{e}$ found as a result of the calculations, is

$$
t \partial_{t}+x_{i} \partial_{x_{i}}-\dot{\rho} \partial_{\dot{\rho}}
$$

The part $-\dot{\rho} \partial_{\dot{\rho}}$ is obtained from $X_{11}^{e}$ using the prolongation formulae. The symmetry operators $X_{j}^{e}(1 \leq j \leq 10)$ are symmetries of the Galilean group ${ }^{3}$, which are independent of a potential function $W(\rho, \dot{\rho})$. The symmetries corresponding to the operators $X_{1}^{e}, X_{2}^{e}, X_{3}^{e}$ are the space translation symmetries, $X_{4}^{e}, X_{5}^{e}, X_{6}^{e}$ are the Galilean boosts, $X_{7}^{e}, X_{8}^{e}$ and $X_{9}^{e}$ are the rotations

\footnotetext{
${ }^{2}$ In the classical approach [7, Chapter 2, Section 6.4] for an equivalence Lie group it is assumed $\xi_{\phi_{k}}^{i}=\zeta_{\phi_{k}}^{j}=0$. Discussion of the generalization of the classical approach is given in [19, Chapter 5, Section 2.1].

${ }^{3}$ This group is admitted by many systems of partial differential equations applied in Newtonian continuum mechanics. See, for example, $[7,8]$ and references therein.
} 
and $X_{10}^{e}$ is the time translation symmetry. The operator $X_{11}^{e}$ corresponds to a scaling symmetry, which is also admitted by the gas dynamics equations [7]. The symmetry corresponding to the operator $X_{16}^{e}$ applies for a gas with a special state equation [7]. Since the equivalence transformations corresponding to the operators $X_{11}^{e}, X_{12}^{e}, \ldots, X_{16}^{e}$ are applied for simplifying the function $W$ in the process of the classification, let us present these transformations. As the function $W$ depends on $\rho$ and $\dot{\rho}$, only the transformations of these variables are presented:

$\begin{array}{llll}X_{11}^{e}: & \rho^{\prime}=\rho, & \dot{\rho}^{\prime}=e^{-a} \dot{\rho}, & W^{\prime}=W ; \\ X_{12}^{e}: & \rho^{\prime}=\rho, & \dot{\rho}^{\prime}=\dot{\rho}, & W^{\prime}=W+a ; \\ X_{13}^{e}: & \rho^{\prime}=\rho, & \dot{\rho}^{\prime}=\dot{\rho}, & W^{\prime}=W+a \rho ; \\ X_{14}^{e}: & \rho^{\prime}=\rho, & \dot{\rho}^{\prime}=\dot{\rho}, & W^{\prime}=W+a \dot{\rho} ; \\ X_{15}^{e}: & \rho^{\prime}=e^{a} \rho, & \dot{\rho}^{\prime}=e^{a} \dot{\rho}, & W^{\prime}=e^{a} W ; \\ X_{16}^{e}: \quad \rho^{\prime}=e^{-2 a} \rho, & \dot{\rho}^{\prime}=e^{-2 a} \dot{\rho}, & W^{\prime}=W .\end{array}$

Here $a$ is the group parameter.

\section{$3 \quad$ Admitted Lie group of (1)}

An admitted generator $X$ of equations (1) is sought in the form

$$
X=\xi^{x_{1}} \partial_{x_{1}}+\xi^{x_{2}} \partial_{x_{2}}+\xi^{x_{3}} \partial_{x_{3}}+\xi^{t} \partial_{t}+\zeta^{u_{1}} \partial_{u_{1}}+\zeta^{u_{2}} \partial_{u_{2}}+\zeta^{u_{3}} \partial_{u_{3}}+\zeta^{\rho} \partial_{\rho},
$$

where the coefficients of the generator are functions of the variables $x_{1}, x_{2}, x_{3}, t, u_{1}, u_{2}, u_{3}, \rho$.

Calculations showed that

$$
\begin{aligned}
& \xi^{x_{1}}=c_{6} x_{1} t+c_{4} t+c_{3} x_{3}+x_{1} c_{7}+x_{1} c_{1}+c_{5}, \\
& \xi^{x_{2}}=c_{6} x_{2} t+c_{12} t+x_{3} c_{11}+x_{2} c_{7}+x_{2} c_{1}-x_{1} c_{12}+c_{13}, \\
& \xi^{x_{3}}=c_{6} x_{3} t+c_{16} t+c_{7} x_{3}+c_{1} x_{3}-c_{11} x_{2}-c_{3} x_{1}+c_{17}, \\
& \xi^{t}=c_{6} t^{2}+c_{7} t+c_{8}, \quad \zeta^{\rho}=\left(-3 c_{6} t+c_{15}\right) \rho, \\
& \zeta^{u_{1}}=c_{3} u_{3}+c_{2} u_{2}-c_{6} u_{1} t+c_{1} u_{1}+c_{6} x_{1}+c_{4}, \\
& \zeta^{u_{2}} c_{11} u_{3}-c_{6} u_{2} t+c_{1} u_{2}-c_{2} u_{1}+c_{6} x_{2}+c_{12}, \\
& \zeta^{u_{3}}=-c_{6} u_{3} t+c_{1} u_{3}-c_{11} u_{2}-c_{3} u_{1}+c_{6} x_{3}+c_{16},
\end{aligned}
$$

where the constants $c_{i}(i=1,2, \ldots, 8,11,12,13,15)$ satisfy the conditions

$$
\begin{aligned}
& 27 c_{6} \rho^{3}\left(3 W_{\dot{\rho} \rho \rho \rho} \dot{\rho} \rho+W_{\dot{\rho} \rho \rho} \dot{\rho}-3 W_{\rho \rho \rho} \rho-W_{\rho \rho}\right)+600 W_{\dot{\rho} \dot{\rho}} c_{6} \dot{\rho}^{2} \rho \\
& +25 \dot{\rho}^{3}\left(5 W_{\dot{\rho} \dot{\rho} \dot{\rho} \dot{\rho}} \dot{\rho}^{2}\left(c_{15}-c_{7}\right)+5 W_{\dot{\rho} \dot{\rho} \dot{\rho} \rho} \dot{\rho} \rho c_{15}+18 W_{\dot{\rho} \dot{\rho} \rho} \rho c_{15}\right. \\
& \left.+W_{\dot{\rho} \dot{\rho} \dot{\rho}} \dot{\rho}\left(28 c_{15}-33 c_{7}-10 c_{1}\right)+18 W_{\dot{\rho} \dot{\rho}}\left(c_{15}-2 c_{7}-2 c_{1}\right)\right)=0, \\
& W_{\dot{\rho} \dot{\rho} \dot{\rho}} \dot{\rho}\left(c_{7}-c_{15}\right)-c_{15} \rho W_{\dot{\rho} \dot{\rho} \rho}+\left(2 c_{1}-c_{15}+2 c_{7}\right) W_{\dot{\rho} \dot{\rho}}+3 c_{6} W_{\dot{\rho} \dot{\rho} \dot{\rho} \rho}=0 \text {, }
\end{aligned}
$$

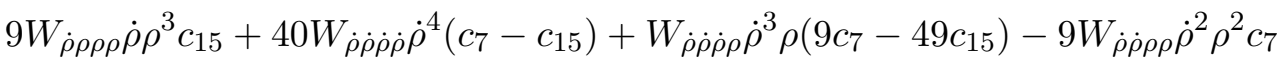

$$
\begin{aligned}
& +8 W_{\dot{\rho} \dot{\rho} \dot{\rho}} \dot{\rho}^{3}\left(10 c_{1}-17 c_{15}+22 c_{7}\right)+2 W_{\dot{\rho} \dot{\rho} \rho} \dot{\rho}^{2} \rho\left(9 c_{1}-37 c_{15}+9 c_{7}\right)-9 W_{\rho \rho \rho} \rho^{3} c_{15} \\
& +9 W_{\dot{\rho} \rho \rho} \dot{\rho} \rho^{2}\left(c_{15}-2 c_{1}\right)+56 W_{\dot{\rho} \dot{\rho}} \dot{\rho}^{2}\left(2 c_{1}-c_{15}+2 c_{7}\right)+9 W_{\rho \rho} \rho^{2}\left(2 c_{1}-c_{15}\right)=0 \text {, } \\
& c_{6}\left(5 W_{\dot{\rho} \dot{\rho} \dot{\rho} \dot{\rho}}+3 W_{\dot{\rho} \dot{\rho} \rho} \rho+5 W_{\dot{\rho} \dot{\rho}}\right)=0 .
\end{aligned}
$$

The determining equations (2)-(5) define the kernel of admitted Lie algebras and its extensions. The kernel of admitted Lie algebras consists of the generators

$$
Y_{1}=\partial_{x_{1}}, \quad Y_{2}=\partial_{x_{2}}, \quad Y_{3}=\partial_{x_{3}}, \quad Y_{10}=\partial_{t},
$$




$$
\begin{aligned}
& Y_{4}=t \partial_{x_{1}}+\partial_{u_{1}}, \quad Y_{5}=t \partial_{x_{2}}+\partial_{u_{2}}, \quad Y_{6}=t \partial_{x_{3}}+\partial_{u_{3}}, \\
& Y_{7}=x_{2} \partial_{x_{3}}-x_{3} \partial_{x_{2}}+u_{2} \partial_{u_{3}}-u_{3} \partial_{u_{2}}, \\
& Y_{8}=x_{3} \partial_{x_{1}}-x_{1} \partial_{x_{3}}+u_{3} \partial_{u_{1}}-u_{1} \partial_{u_{3}}, \\
& Y_{9}=x_{1} \partial_{x_{2}}-x_{2} \partial_{x_{1}}+u_{1} \partial_{u_{2}}-u_{2} \partial_{u_{1}} .
\end{aligned}
$$

Extensions of the kernel depend on the value of the function $W(\rho, \dot{\rho})$. They can only be operators of the form

$$
c_{1} X_{1}+c_{6} X_{6}+c_{7} X_{7}+c_{15} X_{14},
$$

where

$$
\begin{aligned}
& X_{1}=x_{i} \partial_{x_{i}}+u_{i} \partial_{u_{i}}, \quad X_{6}=t\left(t \partial_{t}+x_{i} \partial_{x_{i}}-u_{i} \partial_{u_{i}}-3 \rho \partial_{\rho}\right)+x_{i} \partial_{u_{i}} \\
& X_{7}=x_{i} \partial_{x_{i}}+t \partial t, \quad X_{9}=x_{2} \partial_{x_{2}}+u_{2} \partial_{u_{2}}, \quad X_{14}=\rho \partial_{\rho} .
\end{aligned}
$$

Relations between the constants $c_{1}, c_{6}, c_{7}, c_{15}$ depend on the function $W(\rho, \dot{\rho})$.

\subsection{Case $c_{6} \neq 0$}

Let $c_{6} \neq 0$, then equation (5) gives

$$
5 W_{\dot{\rho} \dot{\rho} \dot{\rho} \dot{\rho}}+3 W_{\dot{\rho} \dot{\rho} \rho} \rho+5 W_{\dot{\rho} \dot{\rho}}=0 .
$$

The general solution of this equation is $W_{\dot{\rho} \dot{\rho}}=\rho^{-5 / 3} g\left(\dot{\rho} \rho^{-5 / 3}\right)$, where the function $g$ is an arbitrary function of integration. Substitution of $W_{\dot{\rho} \dot{\rho}}$ into equation (3) shows that the function $g=2 q_{0}$ is constant. Hence,

$$
W=q_{0} \dot{\rho}^{2} \rho^{-5 / 3}+\varphi_{1}(\rho) \dot{\rho}+\varphi_{2}(\rho),
$$

where the functions $\varphi_{2}(\rho)$ and $\varphi_{1}(\rho)$ are arbitrary. Substituting this potential function in the other equations (2)-(4), one obtains

$$
3 \rho \varphi_{2}^{\prime \prime \prime}+\varphi_{2}^{\prime \prime}=0, \quad\left(c_{7}+2 c_{1}\right) \varphi_{2}^{\prime \prime}=0, \quad c_{15}=-3\left(c_{1}+c_{7}\right) .
$$

If $\varphi_{2}^{\prime \prime}=0$, then the extension of the kernel of admitted Lie algebras is given by the generators

$$
X_{6}, \quad X_{1}-3 X_{14}, \quad X_{7}-3 X_{14} .
$$

If $\varphi_{2}^{\prime \prime}=C_{2} \rho^{-3} \neq 0$, then the extension of the kernel is given by the generators

$$
X_{6}, \quad X_{1}-2 X_{7}+3 X_{14} \text {. }
$$

\subsection{Case $c_{6}=0$}

Let $c_{6}=0$, then equation (3) becomes

$$
-c_{15} a+\left(c_{1}+c_{7}\right) b+c_{7} c=0,
$$

where

$$
a=\dot{\rho} W_{\dot{\rho} \dot{\rho} \dot{\rho}}+\rho W_{\dot{\rho} \dot{\rho} \rho}+W_{\dot{\rho} \dot{\rho}}, \quad b=2 W_{\dot{\rho} \dot{\rho},}, \quad c=\dot{\rho} W_{\dot{\rho} \dot{\rho} \dot{\rho}} .
$$

Further analysis of the determining equations (2)-(4) is similar to the group classification of the gas dynamics equations [7].

Let us analyze the vector space $\operatorname{Span}(V)$, where the set $V$ consists of vectors $(a, b, c)$ with $\rho$ and $\dot{\rho}$ are changed. If the function $W(\rho, \dot{\rho})$ is such that $\operatorname{dim}(\operatorname{Span}(V))=3$, then equation (6) is only satisfied for

$$
c_{1}=0, \quad c_{7}=0, \quad c_{15}=0,
$$

which does not give extensions of the kernel of admitted Lie algebras. Hence, one needs to study $\operatorname{dim}(\operatorname{Span}(V)) \leq 2$. 


\subsubsection{Case $\operatorname{dim}(\operatorname{Span}(V))=2$}

Let $\operatorname{dim}(\operatorname{Span}(V))=2$. There exists a constant vector $(\alpha, \beta, \gamma) \neq 0$, which is orthogonal to the set $V$ :

$$
\alpha a+\beta b+\gamma c=0
$$

This means that the function $W(\rho, \dot{\rho})$ satisfies the equation

$$
(\alpha+\gamma) \dot{\rho} W_{\dot{\rho} \dot{\rho} \dot{\rho}}+\alpha \rho W_{\rho \dot{\rho} \dot{\rho}}=-(\alpha+2 \beta) W_{\dot{\rho} \dot{\rho}} .
$$

The characteristic system of this equation is

$$
\frac{d \dot{\rho}}{(\alpha+\gamma) \dot{\rho}}=\frac{d \rho}{\alpha \rho}=\frac{d W_{\dot{\rho} \dot{\rho}}}{-(\alpha+2 \beta) W_{\dot{\rho} \dot{\rho}}}
$$

The general solution of equation (8) depends on the values of the constants $\alpha, \beta$ and $\gamma$.

Case $\boldsymbol{\alpha}=\mathbf{0}$. Because of equation (7) and the condition $W_{\dot{\rho} \dot{\rho}} \neq 0$, one has $\gamma \neq 0$. The general solution of equation (8) is

$$
W_{\dot{\rho} \dot{\rho}}(\rho, \dot{\rho})=\tilde{\varphi} \dot{\rho}^{k}
$$

where $k=-2 \beta / \gamma$, and $\widetilde{\varphi}$ is an arbitrary function of integration. Substitution of (9) into (6) leads to

$$
c_{15} \rho \tilde{\varphi}^{\prime}-\tilde{\varphi}(\rho)\left(2 c_{1}-(k+1) c_{15}+(k+2) c_{7}\right)=0 .
$$

If $c_{15} \neq 0$, the dimension $\operatorname{dim}(\operatorname{Span}(V))=1$, which contradicts to the assumption. Hence, $c_{15}=0$ and from (10) one obtains $\tilde{c_{1}}=-(k+2) c_{7} / 2$. The extension of the kernel in this case is given by the generator

$$
-p X_{1}+2 X_{7}
$$

where $p=k+2$.

If $(k+2)(k+1) \neq 0$, then integrating (9), one finds

$$
W(\rho, \dot{\rho})=\varphi(\rho) \dot{\rho}^{p}+\varphi_{1}(\rho) \dot{\rho}+\varphi_{2}(\rho),
$$

where $\varphi_{1}(\rho)$ and $\varphi_{2}(\rho)$ are arbitrary functions. Substituting this function $W$ into (2)-(4) one has $\varphi_{2}^{\prime \prime}=0$.

If $k=-2$, then

$$
W(\rho, \dot{\rho})=\varphi(\rho) \ln (\dot{\rho})+\dot{\rho} \varphi_{1}(\rho)+\varphi_{2}(\rho),
$$

and $\varphi_{2}^{\prime \prime}=0$, similar to the previous case.

If $k=-1$, then

$$
W(\rho, \dot{\rho})=\varphi(\rho) \dot{\rho} \ln (\dot{\rho})+\dot{\rho} \varphi_{1}(\rho)+\varphi_{2}(\rho),
$$

and also $\varphi_{2}^{\prime \prime}=0$. 
Case $\boldsymbol{\alpha} \neq \mathbf{0}$. The general solution of equation (9) is

$$
W_{\dot{\rho} \dot{\rho}}(\rho, \dot{\rho})=\varphi\left(\dot{\rho} \rho^{k}\right) \rho^{\lambda},
$$

where $k=-(1+\gamma / \alpha), \lambda=-(1+2 \beta / \alpha)$ and $\varphi$ is an arbitrary function. Substitution of this function into (6) leads to

$$
k_{0} \varphi^{\prime} z+k_{1} \varphi=0
$$

where

$$
z=\dot{\rho} \rho^{k}, \quad k_{0}=c_{7}-c_{15}(k+1), \quad k_{1}=2 c_{1}-c_{15}(\lambda+1)+2 c_{7} .
$$

Since $\operatorname{dim}(\operatorname{Span}(V))=2$, one obtains that $k_{0}=0$ and $k_{1}=0$ or

$$
c_{7}=c_{15}(k+1), \quad c_{1}=c_{15}(p-1) / 2,
$$

where $p=\lambda-2 k$. Integrating (11), one finds

$$
W(\rho, \dot{\rho})=\rho^{p} \varphi\left(\dot{\rho} \rho^{k}\right)+\dot{\rho} \varphi_{1}(\rho)+\varphi_{2}(\rho) .
$$

Substitution of (12) into (2)-(4) gives

$$
\rho \varphi_{2}^{\prime \prime \prime}+(2 k-\lambda+2) \varphi_{2}^{\prime \prime}=0 \text {. }
$$

Solving this equation, one has

$$
\varphi_{2}^{\prime \prime}=C_{2} \rho^{p-2},
$$

where $C_{2}$ is an arbitrary constant. The extension of the kernel is given by the generator

$$
(p-1) X_{1}+2(k+1) X_{7}+2 X_{14} \text {. }
$$

\subsubsection{Case $\operatorname{dim}(\operatorname{Span}(V))=1$}

Let $\operatorname{dim}(\operatorname{Span}(V))=1$. There exists a constant vector $(\alpha, \beta, k) \neq 0$ such that

$$
(a, b, c)=(\alpha, \beta, k) B
$$

with some function $B(\rho, \dot{\rho}) \neq 0$. Because $W_{\dot{\rho} \dot{\rho}} \neq 0$, one has that $\beta \neq 0$. Hence, the function $W(\rho, \dot{\rho})$ satisfies the equations

$$
\dot{\rho} W_{\dot{\rho} \dot{\rho} \dot{\rho}}+\rho W_{\rho \dot{\rho} \dot{\rho}}+(1-2 \tilde{\alpha}) W_{\dot{\rho} \dot{\rho}}=0, \quad \dot{\rho} W_{\dot{\rho} \dot{\rho} \dot{\rho}}-2 \gamma W_{\dot{\rho} \dot{\rho}}=0 .
$$

The general solution of the latter equation is

$$
W_{\dot{\rho} \dot{\rho}}(\rho, \dot{\rho})=\varphi(\rho) \dot{\rho}^{k}
$$

with arbitrary function $\varphi(\rho)$. Substituting this solution into the first equation, one obtains

$$
\rho \varphi^{\prime}(\rho)+(1-2 \tilde{\alpha}+k) \varphi(\rho)=0, \quad \tilde{\alpha}=\alpha / \beta .
$$

Thus,

$$
W_{\dot{\rho} \dot{\rho}}=-q_{0} \dot{\rho}^{k} \rho^{\lambda},
$$


where $\lambda=-(1-2 \tilde{\alpha}+k), q_{0}$ is an arbitrary constant. Since $\operatorname{dim}(\operatorname{Span}(V))=1$, then $q_{0} \neq 0$, $\lambda$ and $k$ are such that $\lambda^{2}+k^{2} \neq 0$.

Substituting (13) into (6), it becomes

$$
-c_{15}(k+\lambda+1)+c_{7}(k+2)+2 c_{1}=0 .
$$

Integration of (13) depends on the quantity of $k$.

If $(k+2)(k+1) \neq 0$, then integrating (13), one obtains

$$
W(\rho, \dot{\rho})=-q_{0} \rho^{\lambda} \dot{\rho}^{p}+\dot{\rho} \varphi_{1}(\rho)+\varphi_{2}(\rho), \quad p(p-1) \neq 0,
$$

where $p=k+2$. Substituting this $W$ into equations (2)-(4), one obtains

$$
\left.c_{1}=\left(c_{15}(p+\lambda-1)-c_{7} p\right)\right) / 2,
$$

with the function $\varphi_{2}(\rho)$ satisfying the condition

$$
c_{15} \rho \varphi_{2}^{\prime \prime \prime}+\varphi_{2}^{\prime \prime}\left(-c_{15}(p+\lambda-2)+c_{7} p\right)=0 .
$$

If $\varphi_{2}^{\prime \prime}=C_{2} \rho^{-\mu} \neq 0$, the extension of the kernel is given by the generator

$$
(1-\mu) X_{1}+2\left(X_{14}+\phi X_{7}\right)
$$

where $\phi=(\mu+\lambda+p-2) / p$. If $\varphi_{2}^{\prime \prime}=0$, the extension is given by the generators

$$
p X_{1}-2 X_{7}, \quad(p+\lambda-1) X_{1}+2 X_{14} .
$$

If $k=-2$, then integrating (13), one obtains

$$
W(\rho, \dot{\rho})=-q_{0} \rho^{\lambda} \ln (\dot{\rho})+\dot{\rho} \varphi_{1}(\rho)+\varphi_{2}(\rho), \quad q_{0} \neq 0 .
$$

Substituting this into equations (2)-(4), we obtain

$$
c_{1}=c_{15}(\lambda-1) / 2,
$$

and the condition

$$
c_{15}\left(\rho \varphi_{2}^{\prime \prime \prime}-\varphi_{2}^{\prime \prime}(\lambda+2)\right)+q_{0} \lambda(\lambda-1)\left(c_{15}-c_{7}\right) \rho^{\lambda-2}=0 .
$$

If $\lambda(\lambda-1)=0$ and $\varphi_{2}$ is arbitrary, then the extension is given only by the generator

$$
X_{7} \text {. }
$$

If $\lambda(\lambda-1)=0$ and $\varphi_{2}^{\prime \prime}=C_{2} \rho^{\lambda+2}$, then the extension of the kernel consists of the generators

$$
(\lambda-1) X_{1}+2 X_{14}, \quad X_{7}
$$

If $\lambda(\lambda-1) \neq 0$ and $\varphi_{2}^{\prime \prime}=C_{2} \rho^{\lambda+2}-\frac{q_{0}}{4} \lambda(\lambda-1) \mu \rho^{\lambda-2}$, then the extension is

$$
(\lambda-1) X_{1}+2\left(X_{14}+(\mu+1) X_{7}\right),
$$

where $c_{7}=(\mu+1) c_{15}$.

If $k=-1$, then integrating (13), one obtains

$$
W(\rho, \dot{\rho})=-q_{0} \rho^{\lambda} \dot{\rho} \ln (\dot{\rho})+\dot{\rho} \varphi_{1}(\rho)+\varphi_{2}(\rho),
$$


and substituting it into equations (2)-(4), we obtain

$$
c_{1}=\left(c_{15} \lambda-c_{7}\right) / 2
$$

and the condition

$$
c_{15} \rho \varphi_{2}^{\prime \prime \prime}+\varphi_{2}^{\prime \prime}\left(-c_{15} \lambda+c_{15}+c_{7}\right)=0 .
$$

One needs to study two cases. If $\varphi_{2}^{\prime \prime} \neq 0$, then the extension is possible only for $\varphi_{2}=C_{2} \rho^{-\mu} \neq 0$, where $\mu=-\lambda+1+c_{7} / c_{15}$. The extension of the kernel is given by the generator

$$
(1-\mu) X_{1}+2(\mu+\lambda-1) X_{7}+2 X_{14} .
$$

If $\varphi_{2}^{\prime \prime}=0$, then the extension of the kernel consists of the generators

$$
X_{1}-2 X_{7}, \quad X_{14}+\lambda X_{7} .
$$

\subsubsection{Case $\operatorname{dim}(\operatorname{Span}(V))=0$}

Let $\operatorname{dim}(\operatorname{Span}(V))=0$. The vector $(a, b, c)$ is constant:

$$
(a, b, c)=(\alpha, \beta, k)
$$

with some constant values $\alpha, \beta$ and $k$. This leads to

$$
W_{\dot{\rho} \dot{\rho}}=-2 q_{0},
$$

where $q_{0} \neq 0$ is constant. Integrating this equation, one obtains

$$
W(\rho, \dot{\rho})=-q_{0} \dot{\rho}^{2}+\dot{\rho} \varphi_{1}(\rho)+\varphi_{2}(\rho) .
$$

Substituting (14) into equation (2)-(4), we obtain

$$
c_{1}=\left(c_{15}-2 c_{7}\right) / 2,
$$

and the condition

$$
c_{15} \rho \varphi_{2}^{\prime \prime \prime}+2 c_{7} \varphi_{2}^{\prime \prime}=0 .
$$

If $\varphi_{2}^{\prime \prime} \neq 0$, then $\varphi_{2}=C_{2} \rho^{-\mu}$, where $\mu=2 c_{7} / c_{15}$. The extension of the kernel consists of the generator

$$
(1-\mu) X_{1}+2 X_{14}+\mu X_{7} .
$$

If $\varphi_{2}^{\prime \prime}=0$, then the extension of the kernel is given by the generators

$$
X_{1}+2 X_{14}, \quad X_{1}-X_{7}
$$

The result of group classification of equations (1) is summarized in Table 1. The linear part with respect to $\dot{\rho}$ of the function $W(\rho, \dot{\rho})$ is omitted. Notice also that the change $t \rightarrow-t$ has to conserve the potential function $W$, this leads to $\varphi_{1}(\rho)=0$.

Remark 1. The Green-Naghdi model belongs to the class $M_{7}$ in Table 1 with $\lambda=1, p=2$ and $\mu=0$. Invariant solutions of the one-dimensional Green-Naghdi model completely studied in $[21]$.

Remark 2. The one-velocity dissipation-free Iordanski-Kogarko-Wijngaarden model has an extension of the kernel of admitted Lie algebras only for a special internal energy of the gas phase (class $M_{3}(p=2)$ in Table 1$)$, which corresponds to a Chaplygin gas $\varepsilon_{20}\left(\rho_{20}\right)=\gamma_{1} / \rho_{20}+\gamma_{0}$, where $\gamma_{1}$ and $\gamma_{0}$ are constants. 
Table 1. Group classification of equations (1).

\begin{tabular}{|l|l|l|l|}
\hline & $W(\rho, \dot{\rho})$ & Extensions & Remarks \\
\hline$M_{1}$ & $-q_{0} \rho^{-5 / 3} \dot{\rho}^{2}+\varphi_{2}(\rho)$ & $X_{6}, X_{1}-2 X_{7}+3 X_{14}$ & $\varphi_{2}^{\prime \prime}=C_{2} \rho^{-3} \neq 0$ \\
\hline$M_{2}$ & & $X_{6}, X_{1}-3 X_{14}, X_{7}-3 X_{14}$ & $\varphi_{2}^{\prime \prime}=0$ \\
\hline$M_{3}$ & $\varphi(\rho) \dot{\rho}^{p}+\varphi_{2}$ & $-p X_{1}+2 X_{7}$ & $\varphi_{2}^{\prime \prime}=0$ \\
\hline$M_{4}$ & $\varphi(\rho) \ln \dot{\rho}+\varphi_{2}$ & $X_{7}$ & $\varphi_{2}^{\prime \prime}=0$ \\
\hline$M_{5}$ & $\dot{\rho} \varphi(\rho) \ln \dot{\rho}+\varphi_{2}$ & $X_{1}-2 X_{7}$ & $\varphi_{2}^{\prime \prime}=0$ \\
\hline$M_{6}$ & $\rho^{p} \varphi\left(\dot{\rho} \rho^{k}\right)+\varphi_{2}$ & $(p-1) X_{1}+2\left(X_{7}(k+1)+X_{14}\right)$ & $\varphi_{2}^{\prime \prime}=C_{2} \rho^{p-2}$ \\
\hline$M_{7}$ & $-q_{0} \rho^{\lambda} \dot{\rho}^{p}+\varphi_{2}$ & $(1-\mu) X_{1}+2\left(X_{14}+\phi X_{7}\right)$ & $\varphi_{2}^{\prime \prime}=C_{2} \rho^{-\mu} \neq 0$, \\
& & & $p(p-1) \neq 0$, \\
& & $p X_{1}-2 X_{7}$, & $(\mu+\lambda+p-2) / p$ \\
\hline$M_{8}$ & & $(p+\lambda-1) X_{1}+2 X_{14}$ & $\begin{array}{l}\varphi_{2}^{\prime \prime}=0, \\
p(p-1) \neq 0\end{array}$ \\
\hline$M_{9}$ & $-q_{0} \rho^{\lambda} \ln \dot{\rho}+\varphi_{2}$ & $X_{7}$ & $\varphi_{2}(\rho)$ arbitrary, \\
& & & $\lambda(\lambda-1)=0$ \\
\hline$M_{10}$ & & $(\lambda-1) X_{1}+2 X_{14}$, & $\varphi_{2}^{\prime \prime}=C_{2} \rho^{\lambda+2}$, \\
& & $X_{7}(\lambda-1)=0$ \\
\hline$M_{11}$ & & $(\lambda-1) X_{1}+2\left(X_{14}+(\mu+1) X_{7}\right)$ & $\varphi_{2}^{\prime \prime}=C_{2} \rho^{\lambda+2}$ \\
& & & $-\frac{q_{0}}{4} \lambda(\lambda-1) \mu \rho^{\lambda-2}$, \\
& & $(1-\mu) X_{1}+2(\mu+\lambda-1) \neq 0$ \\
\hline$M_{12}$ & $-q_{0} \rho^{\lambda} \dot{\rho} \ln \dot{\rho}+\varphi_{2}$ & $X_{1}+2 X_{14}, X_{1}-X_{7}$ & $\varphi_{2}, X_{14}+\lambda X_{7}$ \\
\hline$M_{13}$ & & & $\varphi_{2}^{\prime \prime}=0$ \\
\hline$M_{14}$ & $-q_{0} \rho^{2}+\varphi_{2}$ & & $\varphi_{2}=C_{2} \rho^{-\mu} \neq 0$ \\
\hline$M_{15}$ & & & \\
\hline
\end{tabular}

\section{Special vortex}

In this section a special vortex solution is considered. With the spherical coordinates [12]:

$$
\begin{aligned}
& x=r \sin \theta \cos \varphi, \quad y=r \sin \theta \sin \varphi, \quad z=r \cos \theta, \\
& U=u \sin \theta \cos \varphi+v \sin \theta \sin \varphi+w \cos \theta, \\
& U_{2}=u \cos \theta \cos \varphi+v \cos \theta \sin \varphi-w \sin \theta \\
& U_{3}=-u \sin \varphi+v \cos \varphi
\end{aligned}
$$

the generators $X_{7}, X_{8}, X_{9}$ are

$$
\begin{aligned}
& X_{7}=-\sin \varphi \partial_{\theta}-\cos \varphi \cot \theta \partial_{\varphi}+\cos \varphi(\sin \theta)^{-1}\left(U_{2} \partial_{U_{3}}-U_{3} \partial_{U_{2}}\right), \\
& X_{8}=-\cos \varphi \partial_{\theta}-\sin \varphi \cot \theta \partial_{\varphi}+\sin \varphi(\sin \theta)^{-1}\left(U_{2} \partial_{U_{3}}-U_{3} \partial_{U_{2}}\right), \quad X_{9}=\partial_{\varphi} .
\end{aligned}
$$

Introducing cylindrical coordinates $(H, \omega)$ into the two-dimensional space of vectors $\left(U_{2}, U_{3}\right)$

$$
U_{2}=H \cos \omega, \quad U_{3}=H \sin \omega,
$$

the first two generators become

$$
X_{7}=-\sin \varphi \partial_{\theta}-\cos \varphi \cot \theta \partial_{\varphi}+\cos \varphi(\sin \theta)^{-1} \partial_{\omega},
$$




$$
X_{8}=-\cos \varphi \partial_{\theta}-\sin \varphi \cot \theta \partial_{\varphi}+\sin \varphi(\sin \theta)^{-1} \partial_{\omega}
$$

The singular vortex solution [12] is defined by the representation

$$
U=U(t, r), \quad H=H(t, r), \quad \rho=\rho(t, r), \quad \omega=\omega(t, r, \theta, \varphi) .
$$

The function $\omega(t, r, \theta, \varphi)$ is "superfluous": it depends on all independent variables. If $H=0$, then the tangent component of the velocity vector is equal to zero. This corresponds to the spherically symmetric flows. For a singular vortex, it is assumed that $H \neq 0$.

In a manner similar to [12] one finds that for system (1), the invariant functions $U(t, r)$, $H(t, r)$ and $\rho(t, r)$ have to satisfy the system of partial differential equations with the two independent variables $t$ and $r$ :

$$
\begin{aligned}
& r^{2} D_{0} \rho+\rho\left(r^{2} U\right)_{r}=\rho \alpha h, \quad D_{0} U+\rho^{-1} p_{r}=r^{-3} \alpha^{2}, \\
& D_{0} h=r^{-2} \alpha\left(h^{2}+1\right), \quad D_{0} \alpha=0, \\
& p=\rho\left(W_{\rho}-\dot{\rho} W_{\rho \dot{\rho}}-W_{\dot{\rho} \dot{\rho}} D_{0} \dot{\rho}\right)+W_{\dot{\rho}} \dot{\rho}-W,
\end{aligned}
$$

where $\alpha=r H, D_{0}=\partial_{t}+U \partial_{r}$, and the function $h(t, r)$ is introduced for convenience during the compatibility analysis.

The equivalence Lie group of equations (15) corresponds to the generators

$$
\begin{array}{ll}
X_{0}^{e}=\partial_{t}, \quad X_{2}^{e}=\rho \partial_{W}, \quad X_{3}^{e}=2 t \partial_{t}-U \partial_{U}-3 \rho \partial_{\rho}-5 \dot{\rho} \partial_{\dot{\rho}}-3 W \partial_{W}, \\
X_{4}^{e}=\dot{\rho} \partial_{\dot{\rho}}+\rho \partial_{\rho}+W \partial_{W}, & X_{5}^{e}=x \partial_{x}+U \partial_{U}+2 \alpha \partial_{\alpha}+2 W \partial_{W} .
\end{array}
$$

Calculations yield that the kernel of admitted Lie algebras consists of the generator

$$
X_{0}=\partial_{t},
$$

extensions of the kernel can only be operators of the form

$$
k_{1} X_{1}+k_{2} X_{2}+k_{3} X_{3}+k_{4} X_{4}
$$

where

$$
\begin{aligned}
& X_{1}=t \partial_{t}-U \partial_{U}-\alpha \partial_{\alpha}+\dot{\rho} \partial_{\dot{\rho}}, \quad X_{2}=t\left(t \partial_{t}+r \partial_{r}-U \partial_{U}-3 \rho \partial_{\rho}-5 \dot{\rho} \partial_{\dot{\rho}}\right)+r \partial_{U}-3 \rho \partial_{\dot{\rho}}, \\
& X_{3}=2 t \partial_{t}+r \partial_{r}-U \partial_{U}-3 \rho \partial_{\rho}-5 \dot{\rho} \partial_{\dot{\rho}}, \quad X_{4}=\dot{\rho} \partial_{\dot{\rho}}+\rho \partial_{\rho} .
\end{aligned}
$$

The constants $k_{i}(i=1,2,3,4)$ depend on the function $W(\rho, \dot{\rho})$. These extensions are presented in Table 2.

\subsection{Steady-state special vortex}

Let us consider the invariant solution corresponding to the kernel $\left\{X_{0}\right\}$. This type of solution for the gas dynamics equations was studied in [14]. The representation of the solution is

$$
\rho=\rho(r), \quad U=U(r), \quad h=h(r), \quad \alpha=\alpha(r) .
$$

Equations (15) become

$$
\begin{aligned}
& U \rho^{\prime}+\rho\left(r^{2} U\right)^{\prime}=\rho \alpha h, \quad U U^{\prime}+\rho^{-1} p^{\prime}=r^{-3} \alpha^{2}, \\
& U h^{\prime}=r^{-2} \alpha\left(h^{2}+1\right), \quad U \alpha^{\prime}=0, \\
& p=\rho\left(W_{\rho}-U \rho^{\prime} W_{\rho \dot{\rho}}-W_{\dot{\rho} \dot{\rho}} U\left(U \rho^{\prime}\right)^{\prime}\right)+W_{\dot{\rho}} U \rho^{\prime}-W, \quad \dot{\rho}=U \rho^{\prime} .
\end{aligned}
$$


Table 2. Group classification of equations (15).

\begin{tabular}{|l|l|l|l|}
\hline & $W(\rho, \dot{\rho})$ & Extensions & Remarks \\
\hline$M_{1}$ & $-q_{0} \dot{\rho}^{2} \rho^{-5 / 3}+\beta \rho^{5 / 3}$ & $X_{2}, X_{3}$ & $q_{0} \beta \neq 0$ \\
\hline$M_{2}$ & $-q_{0} \dot{\rho}^{2} \rho^{-5 / 3}$ & $X_{2}, X_{1}, X_{3}$ & $q_{0} \neq 0$ \\
\hline$M_{3}$ & $\varphi(\rho) \dot{\rho}^{p}$ & $X_{1}+(2-p) X_{3}$ & $p(p-1) \neq 0$ \\
\hline$M_{4}$ & $-\left(q_{0} \rho+\gamma\right) \ln (\dot{\rho})+\varphi_{2}(\rho)$ & $X_{1}+X_{3}$ & $\varphi_{2}$ arbitrary \\
\hline$M_{5}$ & $\varphi(\rho) \dot{\rho} \ln (\dot{\rho})$ & $2 X_{1}+X_{3}$ & \\
\hline$M_{6}$ & $\rho^{\lambda} \varphi\left(\dot{\rho} \rho^{k}\right)+\varphi_{2}(\rho)$ & $2 X_{1}-(\lambda-2) X_{3}, X_{4}-k X_{3}$ & $\varphi_{2}^{\prime \prime}=C_{2} \rho^{\lambda-2}$ \\
\hline$M_{7}$ & $-q_{0} \rho^{\lambda} \dot{\rho}^{p}+\varphi_{2}(\rho)$ & $2\left(\mu X_{1}+2(2 \mu+p(\lambda-\mu)) X_{3}\right.$ & $\varphi_{2}^{\prime \prime}=C_{2} \rho^{\mu}$ \\
& & $+(2-\lambda)\left(2 X_{1}+(2-p) X_{3}\right)$ & $p(p-1) \neq 0$ \\
\hline$M_{8}$ & $-q_{0} \rho^{\lambda} \dot{\rho}^{p}$ & $-2 X_{1}+(2-\lambda) X_{3}$, & $p(p-1) \neq 0$ \\
\hline$M_{9}$ & $-q_{0} \rho^{\lambda} \ln (\dot{\rho})+\varphi_{2}(\rho)$ & $(p-2) X_{3}-2 X_{6}$ & $X_{1}+X_{3}$ \\
\hline$M_{10}$ & & & $\varphi_{2}$ arbitrary \\
\hline & & $X_{3}+X_{6}, 2 X_{1}+(\lambda-1) X_{3}$ & $\varphi_{2}^{\prime \prime}=C_{2} \rho^{\lambda-2}$ \\
\hline$M_{11}$ & & $X_{1}+\frac{\lambda-1}{2} X_{3}+X_{6}$ & $\lambda(\lambda-1)=0$ \\
\hline$M_{12}$ & $-q_{0} \rho^{\lambda} \dot{\rho} \ln (\dot{\rho})+\varphi_{2}(\rho)$ & $2 X_{1}+\lambda X_{3}$ & $\varphi_{2}^{\prime \prime}=\rho^{\lambda-2}\left(q_{0} \ln (\rho)+\beta\right)$ \\
& & $+(\lambda-\mu-1)\left(X_{3}+2 X_{6}\right)$ & $\lambda(\lambda-1) \neq 0$ \\
\hline$M_{13}$ & $-q_{0} \rho^{\lambda} \dot{\rho} \ln (\dot{\rho})$ & $2 X_{1}+X_{3}, X_{4}$ & $\varphi_{2}^{\prime \prime}=C_{2} \rho^{\mu} \neq 0$ \\
\hline$M_{14}$ & $-q_{0} \dot{\rho}^{2}+\varphi_{2}(\rho)$ & $2 X_{1}+X_{3}-\mu X_{6}$ & \\
\hline$M_{15}$ & $-q_{0} \dot{\rho}^{2}$ & $X_{1}, X_{2}$ & $\varphi_{2}^{\prime \prime}=C_{2} \rho^{\mu} \neq 0$ \\
\hline
\end{tabular}

In [14] it is shown that for the gas dynamics equations all dependent variables can be represented through the function $h(r)$, which satisfies a first-order ordinary differential equation. Here also all dependent variables can be defined through the function $h(r)$, but the equation for $h(r)$ is a fourth-order ordinary differential equation. In fact, since $H \neq 0$, from (16) one obtains that $U \neq 0$. Hence, $\alpha=\alpha_{0}$, where $\alpha_{0}$ is constant. From the first and third equations of (16), one finds

$$
\rho=R_{0} \frac{h^{\prime}}{\sqrt{h^{2}+1}}, \quad U=\frac{\alpha_{0}\left(h^{2}+1\right)}{h^{\prime}} .
$$

In this case

$$
\dot{\rho}=-\alpha_{0} R_{0} h^{\prime}\left(\frac{\sqrt{h^{2}+1}}{h^{\prime}}\right)^{\prime}
$$

and after substituting $\rho$ and $\dot{\rho}$ into the formula for the pressure, one has

$$
p=F\left(h, h^{\prime}, h^{\prime \prime}, h^{\prime \prime \prime}\right),
$$

where the function $F$ is defined by the potential function $W$. Substituting representations of $\rho, U$ and $p$ into the second equation of (15), one obtains the fourth-order ordinary differential equation for the function $h(r)$. 


\subsection{Invariant solutions of (15) with $W=-q_{0} \dot{\rho}^{2} \rho^{-5 / 3}+\beta \rho^{5 / 3}$}

System of equations (15) with the potential function

$$
W=-q_{0} \dot{\rho}^{2} \rho^{-5 / 3}+\beta \rho^{5 / 3}
$$

admit the Lie group corresponding to the Lie algebra $L_{3}=\left\{X_{0}, X_{2}, X_{3}\right\}$.

If $\beta=0$, then there is one more admitted generator $X_{1}$. The four-dimensional Lie algebra with the generators $\left\{X_{0}, X_{1}, X_{2}, X_{3}\right\}$ is denoted by $L_{4}$.

The structural constants of the Lie algebra $L_{4}$ are defined by the table of commutators:

\begin{tabular}{c|cccc} 
& $X_{0}$ & $X_{1}$ & $X_{2}$ & $X_{3}$ \\
\hline$X_{0}$ & 0 & $X_{0}$ & $X_{3}$ & $2 X_{0}$ \\
$X_{1}$ & & 0 & $X_{2}$ & 0 \\
$X_{2}$ & & & 0 & $-2 X_{2}$ \\
$X_{3}$ & & & & 0
\end{tabular}

Solving the Lie equations for the automorphisms, one obtains:

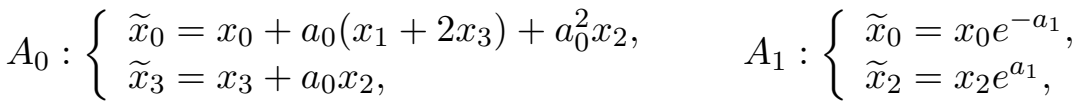

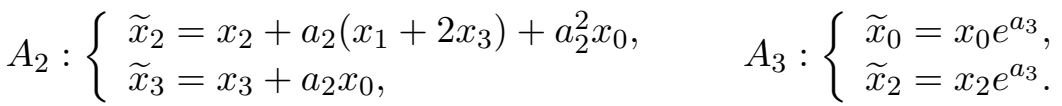

Construction of the optimal system of one-dimensional admitted subalgebras consists of using the automorphisms $A_{i}(i=0,1,2,3)$ for simplifications of the coordinates $\left(x_{0}, x_{1}, x_{2}, x_{3}\right)$ of the generator

$$
X=\sum_{j=0}^{3} x_{j} X_{j} .
$$

Here $k$ is the dimension of the Lie algebra $L_{k}(k=3,4)$. In the case $L_{3}$ one has to assume that the coordinate $x_{1}=0$.

Beside automorphisms for constructing optimal system of subalgebras one can use involutions. Equations (15) posses the involutions $E$, corresponding to the change $t \rightarrow-t$. The involution $E$ acts on the generator

$$
X=\sum_{j=0}^{3} x_{j} X_{j} .
$$

by transforming the generator $X$ into the generator $\widetilde{X}$ with the changed coordinates:

$$
E:\left\{\begin{array}{l}
\widetilde{x}_{0}=-x_{0} \\
\widetilde{x}_{2}=-x_{2} .
\end{array}\right.
$$

Here only the changed coordinates are presented.

\subsection{One-dimensional subalgebras}

One can decompose the Lie algebra $L_{4}$ as $L_{4}=I \oplus N$, where $I=L_{3}$ is an ideal and $N=\left\{X_{1}\right\}$ is a subalgebra of $L_{4}$. Classification of the subalgebra $N=\left\{X_{1}\right\}$ is simple: it consists of the subalgebras:

$$
N_{1}=\{0\}, \quad N_{2}=\left\{X_{1}\right\} .
$$


According to the algorithm [22] for construction of an optimal system of one-dimensional subalgebras one has to consider two types of generators: (a) $X=x_{0} X_{0}+x_{2} X_{2}+x_{3} X_{3}$, (b) $X=X_{1}+x_{0} X_{0}+x_{2} X_{2}+x_{3} X_{3}$. Notice that case (a) corresponds to the Lie algebra $L_{3}$. Hence, classifying the Lie algebra $L_{4}$, one also obtains classification of the Lie algebra $L_{3}$.

\subsubsection{Case (a)}

Assuming that $x_{0} \neq 0$, choosing $a_{2}=-x_{3} / x_{0}$, one maps $x_{3}$ into zero. This means that $\widetilde{x}_{3}=0$. For simplicity of explanation, we write it as $x_{3}\left(A_{2}\right) \rightarrow 0$. In this case $x_{2}\left(A_{2}\right) \rightarrow \widetilde{x}_{2}=x_{2}-x_{3}^{2} / x_{0}$. If $\widetilde{x}_{2} \neq 0$, then applying $x_{2}\left(A_{1}\right) \rightarrow \pm 1$, hence, the generator $X$ becomes

$$
X_{2}+\alpha X_{0}, \quad \alpha= \pm 1 .
$$

If $\widetilde{x}_{2}=0$, then one has the subalgebra: $\left\{X_{0}\right\}$.

In the case $x_{0}=0$, if $x_{3} \neq 0$ or $x_{2} \neq 0$, then, applying $A_{0}$, one can obtain $x_{0} \neq 0$, which leads to the previous case. Hence, without loss of generality one also assumes that $x_{3}=0, x_{2}=0$. Thus, the optimal system of one-dimensional subalgebras in case (a) consists of the subalgebras

$$
\left\{X_{2} \pm X_{0}\right\}, \quad\left\{X_{0}\right\}
$$

This set of subalgebras also composes an optimal system of one-dimensional subalgebras of the algebra $L_{3}$.

\subsubsection{Case (b)}

Assuming that $x_{0} \neq 0$, choosing $a_{2}=-x_{3} / x_{0}$, one maps $x_{3}$ into zero. In this case $x_{2}\left(A_{2}\right) \rightarrow$ $\widetilde{x}_{2}=x_{2}-x_{3}\left(1-x_{3}\right) / x_{0}$. If $\widetilde{x}_{2} \neq 0$, then applying $A_{1}$, and $E_{2}$ (if necessary), one maps the generator $X$ into

$$
X_{1}+X_{2}+\gamma X_{0}
$$

where $\gamma \neq 0$ is an arbitrary constant. If $\widetilde{x}_{2}=0$, then $x_{0}\left(A_{0}\right) \rightarrow 0$, and the generator $X$ becomes $X_{1}$.

In the case $x_{0}=0$, if $2 x_{3}+1 \neq 0$ or $x_{2} \neq 0$, then, applying $A_{0}$, one can obtain $x_{0} \neq 0$, which leads to the previous case. Hence, without loss of generality one also assumes that $x_{3}=-1 / 2$, $x_{2}=0$, and the generator $X$ becomes $X_{3}-2 X_{1}$.

Thus, the optimal system of one-dimensional subalgebras of the Lie algebra $L_{4}$ consists of the subalgebras

$$
\left\{X_{2} \pm X_{0}\right\}, \quad\left\{X_{0}\right\}, \quad\left\{X_{1}+X_{2}+\gamma X_{0}\right\}, \quad\left\{X_{3}-2 X_{1}\right\}, \quad\left\{X_{1}\right\}
$$

where $\gamma \neq 0$ is an arbitrary constant.

Remark 3. An optimal system of subalgebras for $W=-q_{0} \rho^{-3} \dot{\rho}^{2}+\beta \rho^{3}$ with arbitrary $\beta$ consists of the subalgebras (17).

Remark 4. The subalgebra $\left\{X_{2}-X_{0}\right\}$ is equivalent to the subalgebra: $\left\{X_{3}\right\}$.

\subsection{Invariant solutions of $X_{1}+X_{2}+\gamma X_{0}$}

The generator of this Lie group is

$$
X=\gamma X_{0}+X_{1}+X_{2}=\left(t^{2}+t+\gamma\right) \partial_{t}+t r \partial_{r}-3 t \rho \partial_{\rho}+(r-U(t+1)) \partial_{U}-\alpha \partial_{\alpha}
$$


To find invariants, one needs to solve the equation

$$
X J=0,
$$

where $J=J(t, r, \rho, U, \alpha, h)$. A solution of this equation depends on the value of $\gamma$.

Let $\gamma=\mu^{2}+1 / 4$. In this case invariants of the Lie group are

$$
y=r s, \quad V=s\left(\left((t+1 / 2)^{2}+\mu^{2}\right) U-r t\right), \quad R=\rho s^{-3}, \quad \Lambda=\alpha e^{\frac{1}{\mu} \arctan \left(\frac{2 t+1}{2 \mu}\right)}, \quad h,
$$

where

$$
s=\left((t+1 / 2)^{2}+\mu^{2}\right)^{-1 / 2} e^{\frac{1}{2 \mu} \arctan \left(\frac{2 t+1}{2 \mu}\right)} .
$$

The representation of an invariant solution is

$$
s\left(\left((t+1 / 2)^{2}+\mu^{2}\right) U-r t\right)=V(y), \quad \rho=s^{3} R(y), \quad \alpha=\Lambda e^{-\frac{1}{\mu} \arctan \left(\frac{2 t+1}{2 \mu}\right)}, \quad h=h(y) .
$$

Substituting the representation of a solution into (15), one obtains the system of four ordinary differential equations

$$
\begin{aligned}
V^{\prime}= & -\frac{R^{\prime}}{R} V+(\Lambda h-8 V y) /\left(4 y^{2}\right), \quad h^{\prime}=\frac{\Lambda}{V} \frac{\left(h^{2}+1\right)}{4 y^{2}}, \quad \Lambda^{\prime}=\frac{\Lambda}{V}, \\
R^{\prime \prime \prime}= & \left(-\left(\left(8 \left(\left(3(4(44 V+5 y) y-19 \Lambda h) R+308 R^{\prime} V y^{2}\right) R^{\prime 2}\right.\right.\right.\right. \\
& \left.-3\left(88 R^{\prime} V y^{2}-9 \Lambda h R+12(6 V+y) R y\right) R^{\prime \prime} R\right) V q_{0} y-9 R^{2 / 3}(4(4(2 V+y) V \\
& \left.\left.\left.-\left(4 \mu^{2}+1\right) y^{2}\right) y^{2}+(\Lambda-4 h V y) \Lambda\right) R^{3}\right) y-18\left(8\left(R^{2 / 3} V y^{3}+4 \Lambda h q_{0}\right) V y\right. \\
& \left.\left.\left.-\left(2 h^{2}+1\right) \Lambda^{2} q_{0}-4\left(8(5 V+y) V-\left(4 \mu^{2}+1\right) y^{2}\right) q_{0} y^{2}\right) R^{\prime} R^{2}\right)\right) /\left(288 R^{2} V^{2} q_{0} y^{4}\right) .
\end{aligned}
$$

Let $\gamma=-\mu^{2}+1 / 4$. A representation of a solution is

$$
\begin{aligned}
& \left.s\left((t+1 / 2)^{2}-\mu^{2}\right) U-r t\right)=V(y), \quad \alpha(t+1 / 2-\mu)^{\frac{1}{2 \mu}}(t+1 / 2+\mu)^{-\frac{1}{2 \mu}}=\Lambda(y), \\
& \rho(t+1 / 2-\mu)^{3 \alpha_{1}}(t+1 / 2+\mu)^{3 \alpha_{2}}=R(y), \quad h=h(y),
\end{aligned}
$$

where

$$
y=r s, \quad s=(t+1 / 2-\mu)^{-\alpha_{1}}(t+1 / 2+\mu)^{-\alpha_{2}}, \quad \alpha_{1}=\frac{2 \mu-1}{4 \mu}, \quad \alpha_{2}=\frac{2 \mu+1}{4 \mu} .
$$

In this case

$$
\begin{aligned}
V^{\prime}= & -V \frac{R^{\prime}}{R}+\frac{\Lambda h-2 V y)}{y^{2}}, \quad h^{\prime}=\Lambda \frac{\left(h^{2}+1\right)}{V y^{2}}, \quad \Lambda^{\prime}=\frac{\Lambda}{V}, \\
R^{\prime \prime \prime}= & \left(528 R^{\prime \prime} R^{\prime} R V^{2} q_{0} y^{4}+72 R^{\prime \prime} R^{2} V q_{0} y^{2}\left(-3 \Lambda h+6 V y+y^{2}\right)-616 R^{\prime}{ }^{3} V^{2} q_{0} y^{4}\right. \\
& +24 R^{\prime 2} R V q_{0} y^{2}\left(19 \Lambda h-44 V y-5 y^{2}\right)+18 R^{\prime} R^{2}\left(2 R^{2 / 3} V^{2} y^{4}-8 \Lambda^{2} h^{2} q_{0}\right. \\
& \left.-4 \Lambda^{2} q_{0}+32 \Lambda h V q_{0} y-40 V^{2} q_{0} y^{2}-8 V q_{0} y^{3}-4 \mu^{2} q_{0} y^{4}+q_{0} y^{4}\right) \\
& \left.+9 R^{2 / 3} R^{3} y\left(4 \Lambda^{2}-4 \Lambda h V y+8 V^{2} y^{2}+4 V y^{3}+4 \mu^{2} y^{4}-y^{4}\right)\right) /\left(72 R^{2} V^{2} q_{0} y^{4}\right) .
\end{aligned}
$$

Let $\gamma=1 / 4$. A representation of an invariant solution is

$$
s\left((t+1 / 2)^{2} U-r t\right)=V(y), \quad \rho=s^{3} R(y), \quad \alpha=e^{2 /(2 t+1)} \Lambda(y), \quad h=h(y),
$$

where

$$
y=r s, \quad s=\frac{1}{(t+1 / 2)} e^{-1 /(2 t+1)} .
$$


In this case

$$
\begin{aligned}
V^{\prime}= & -V \frac{R^{\prime}}{R}+\frac{(\Lambda h-2 V y)}{y^{2}}, \quad h^{\prime}=\frac{\Lambda}{V} \frac{\left(h^{2}+1\right)}{y^{2}}, \quad \Lambda^{\prime}=\frac{\Lambda}{V}, \\
R^{\prime \prime \prime}= & \left(528 R^{\prime \prime} R^{\prime} R V^{2} q_{0} y^{4}+72 R^{\prime \prime} R^{2} V q_{0} y^{2}\left(-3 \Lambda h+6 V y+y^{2}\right)-616 R^{\prime 3} V^{2} q_{0} y^{4}\right. \\
& +24 R^{\prime 2} R V q_{0} y^{2}\left(19 \Lambda h-44 V y-5 y^{2}\right)+18 R^{\prime} R^{2}\left(2 R^{2 / 3} V^{2} y^{4}-8 \Lambda^{2} h^{2} q_{0}-4 \Lambda^{2} q_{0}\right. \\
& \left.+32 \Lambda h V q_{0} y-40 V^{2} q_{0} y^{2}-8 V q_{0} y^{3}+q_{0} y^{4}\right)+9 R^{2 / 3} R^{3} y\left(4 \Lambda^{2}-4 \Lambda h V y+8 V^{2} y^{2}\right. \\
& \left.\left.+4 V y^{3}-y^{4}\right)\right) /\left(72 R^{2} V^{2} q_{0} y^{4}\right) .
\end{aligned}
$$

These equations were obtained assuming that $V \neq 0$. The case $V=0$ leads to

$$
\Lambda=0, \quad 2 q_{0} R^{\prime}-y R^{5 / 3}=0 .
$$

\subsection{Invariant solutions of $X_{3}-2 X_{1}$}

Invariants of the generator

$$
X_{3}-2 X_{1}=r \partial_{r}-3 \rho \partial_{\rho}+U \partial_{U}+2 \alpha \partial_{\alpha}
$$

are

$$
U=r V(y), \quad \rho=r^{-3} R(y), \quad \alpha=r^{2} \Lambda(y), \quad h=h(y),
$$

where $y=t$. Substitution into equations (15) gives that the functions $V(y), R(y), \Lambda(y)$ and $h(y)$ have to satisfy the equations

$$
\begin{aligned}
& h^{\prime}=\Lambda\left(h^{2}+1\right), \quad \Lambda^{\prime}=-2 \Lambda V, \quad R^{\prime}=\Lambda h R, \\
& 3\left(R^{2 / 3}+6 q_{0}\right)\left(V^{\prime}+V^{2}\right)=\Lambda^{2}\left(4 q_{0}\left(h^{2}-3\right)+3\left(R^{2 / 3}+6 q_{0}\right)\right) .
\end{aligned}
$$

\subsection{Invariant solutions of $X_{1}$}

Invariants of the generator $X_{1}$

$$
X_{1}=t \partial_{t}-U \partial_{U}-\alpha \partial_{\alpha}
$$

are

$$
x, \quad U t, \quad \rho, \quad h, \quad \alpha t \text {. }
$$

An invariant solution has the representation

$$
U=t^{-1} V(y), \quad \rho=R(y), \quad \alpha=t^{-1} \alpha(y), \quad h=h(y),
$$

where $y=x$. Substituting into equations (15), one obtains

$$
\begin{aligned}
V^{\prime}= & -V \frac{R^{\prime}}{R}+\frac{\Lambda h-2 V y}{y^{2}}, \quad h^{\prime}=\frac{\Lambda}{V} \frac{\left(h^{2}+1\right)}{y^{2}}, \quad \Lambda^{\prime}=\frac{\Lambda}{V}, \\
R^{\prime \prime \prime}= & \left(132 R^{\prime \prime} R^{\prime} R V^{2} q_{0} y^{4}+18 R^{\prime \prime} R^{2} V q_{0} y^{2}\left(-3 \alpha h+6 V y+y^{2}\right)-154 R^{\prime 3} V^{2} q_{0} y^{4}\right. \\
& +6 R^{\prime 2} R V q_{0} y^{2}\left(19 \alpha h-44 V y-5 y^{2}\right)+9 R^{\prime} R^{2}\left(R^{2 / 3} V^{2} y^{4}-4 \alpha^{2} h^{2} q_{0}-2 \alpha^{2} q_{0}\right. \\
& \left.+16 \alpha h V q_{0} y-20 V^{2} q_{0} y^{2}-4 V q_{0} y^{3}\right)+9 R^{2 / 3} R^{3} y\left(\alpha^{2}-\alpha h V y\right. \\
& \left.\left.+2 V^{2} y^{2}+V y^{3}\right)\right) /\left(18 R^{2} V^{2} q_{0} y^{4}\right) .
\end{aligned}
$$

Here it is assumed that $V \neq 0$. The case $V=0$ only leads to the condition $\Lambda=0$. 


\subsection{Invariant solutions of $X_{2}+X_{0}$}

$$
X_{2}=t\left(t \partial_{t}+r \partial_{r}-U \partial_{U}-3 \rho \partial_{\rho}\right)+r \partial_{U}
$$

Invariants of the generator

$$
X_{2}+X_{0}=\left(t^{2}+1\right) \partial_{t}+t r \partial_{r}-3 t \rho \partial_{\rho}+(r-t U) \partial_{U}
$$

are

$$
r\left(t^{2}+1\right)^{-1 / 2}, \quad U\left(t^{2}+1\right)^{1 / 2}-r t\left(t^{2}+1\right)^{-1 / 2}, \quad \rho\left(t^{2}+1\right)^{3 / 2}, \quad \alpha, \quad h .
$$

An invariant solution has the representation

$$
U\left(t^{2}+1\right)^{1 / 2}-r t\left(t^{2}+1\right)^{-1 / 2}=V(y), \quad \rho=\left(t^{2}+1\right)^{-3 / 2} R(y), \quad \alpha=\alpha(y), \quad h=h(y) .
$$

where $y=r\left(t^{2}+1\right)^{-1 / 2}$. Substituting into equations (15), one has to study two cases: (a) $V=0$, and (b) $V \neq 0$.

Assuming $V=0$, one obtains that $\Lambda=0$, and the function $R$ satisfies the equation

$$
2\left(5 \beta R^{4 / 3}-9 q_{0}\right) R^{\prime}+9 y R^{5 / 3}=0 .
$$

If $V \neq 0$, then one obtains

$$
\begin{aligned}
V^{\prime}= & -V \frac{R^{\prime}}{R}+\frac{(\Lambda h-2 V y)}{y^{2}}, \quad h^{\prime}=\frac{\Lambda}{V} \frac{\left(h^{2}+1\right)}{y^{2}}, \quad \Lambda^{\prime}=0, \\
R^{\prime \prime \prime}= & \left(132 R^{\prime \prime} R^{\prime} R V^{2} q_{0} y^{4}+54 R^{\prime \prime} R^{2} V q_{0} y^{2}(-\Lambda h+2 V y)-154 R^{\prime 3} V^{2} q_{0} y^{4}\right. \\
& +6 R^{\prime 2} R V q_{0} y^{2}(19 \Lambda h-44 V y)-10 R^{1 / 3} R^{\prime} R^{3} \beta y^{4}+9 R^{\prime} R^{2}\left(R^{2 / 3} V^{2} y^{4}-4 \Lambda^{2} h^{2} q_{0}\right. \\
& \left.-2 \Lambda^{2} q_{0}+16 \Lambda h V q_{0} y-20 V^{2} q_{0} y^{2}+2 q_{0} y^{4}\right)+9 R^{2 / 3} R^{3} y\left(\Lambda^{2}-\Lambda h V y+2 V^{2} y^{2}\right. \\
& \left.\left.-y^{4}\right)\right) /\left(18 R^{2} V^{2} q_{0} y^{4}\right) .
\end{aligned}
$$

\subsection{Invariant solutions of $X_{2}-X_{0}$}

Since the Lie algebra $\left\{X_{2}-X_{0}\right\}$ is equivalent to the Lie algebra with the generator $\left\{X_{3}\right\}$, then for the sake of simplicity an invariant solution with respect to

$$
X_{3}=2 t \partial_{t}+r \partial_{r}-U \partial_{U}-3 \rho \partial_{\rho}
$$

is considered here. Invariants of the generator $X_{3}$ are

$$
r t^{-1 / 2}, \quad U t^{1 / 2}, \quad \rho t^{3 / 2}, \quad h, \quad \alpha .
$$

An invariant solution has the representation

$$
U=t^{-1 / 2} V(y), \quad \rho=t^{-3 / 2} R(y), \quad \alpha=\alpha(y), \quad h=h(y),
$$

where $y=r t^{-1 / 2}$.

Substituting into equations (15), one has to study two cases: (a) $V-y / 2=0$, and (b) $V-y / 2 \neq 0$.

Assuming $V-y / 2=0$, one obtains that $\Lambda=0$, and the function $R$ satisfies the equation

$$
2\left(20 \beta R^{4 / 3}+9 q_{0}\right) R^{\prime}-9 y R^{5 / 3}=0 .
$$


If $V-y / 2 \neq 0$, then one obtains

$$
\begin{aligned}
V^{\prime}= & (y / 2-V) \frac{R^{\prime}}{R}+\frac{2 \Lambda h-(4 V-3 y) y}{2 y^{2}}, \quad h^{\prime}=\frac{\Lambda}{(V-y / 2)} \frac{\left(h^{2}+1\right)}{y^{2}}, \quad \Lambda^{\prime}=0, \\
R^{\prime \prime \prime}= & \left(2(y-2 V)^{2} q_{0} y^{3}\left(66 R^{\prime \prime} R^{\prime} R y+54 R^{\prime \prime} R^{2}-77 R^{\prime 3} y-132 R^{\prime 2} R\right)\right. \\
& +9(y-2 V)^{2} y^{2} R^{2}\left(R^{\prime} R^{2 / 3} y^{2}-20 R^{\prime} q_{0}+2 R^{5 / 3} y\right)-18 R^{\prime} R^{2} y^{4} q_{0} \\
& +6(y-2 V) \alpha h y\left(18 R^{\prime \prime} R^{2} q_{0} y-38 R^{\prime 2} R q_{0} y-48 R^{\prime} R^{2} q_{0}+3 R^{2 / 3} R^{3} y\right) \\
& -72 R^{\prime} \alpha^{2} R^{2} q_{0}\left(2 h^{2}+1\right)-40 R^{10 / 3} R^{\prime} \beta y^{4}+36 R^{2 / 3} \alpha^{2} R^{3} y \\
& \left.+9 R^{11 / 3} y^{5}\right) /\left(18 R^{2} q_{0} y^{4}(y-2 V)^{2}\right) .
\end{aligned}
$$

\section{Conclusion}

In this paper the complete group classification of the three-dimensional equations describing a motion of fluids with internal inertia (1) is given. The classification is considered with respect to the potential function $W(\rho, \dot{\rho})$. Detailed study of one class of partially invariant solutions (the Ovsyannikov vortex) for a particular potential function is presented. This solution is essentially three-dimensional.

\section{Acknowledgments}

The work of P.S. has been supported by scholarship of the Ministry of University Affairs of Thailand. The authors also thank S.L. Gavrilyuk for fruitful discussions, and E. Schulz for his kind help.

\section{References}

[1] Gavrilyuk S.L., Teshukov V.M., Generalized vorticity for bubbly liquid and dispersive shallow water equations, Contin. Mech. Thermodyn. 13 (2001), 365-382.

[2] Iordanski S.V., On the equations of motion of the liquid containing gas bubbles, Prikl. Mekh. Tekhn. Fiz. 3 (1960), 102-111.

[3] Kogarko B.S., On a model of a cavitating liquid, Dokl. Akad. Nauk SSSR 137 (1961), 1331-1333 (English transl.: Soviet Physics Dokl. 6 (1961), 305-306).

[4] van Wijngaarden L., On the equations of motion for mixtures of liquid and gas bubbles, J. Fluid Mech. 33 (1968), 465-474.

[5] Green A.E., Naghdi P.M., A derivation of equations for wave propagation in water of variable depth, J. Fluid Mech. 78 (1976), 237-246.

[6] Salmon R., Lectures on geophysical fluid dynamics, Oxford University Press, New York, 1998.

[7] Ovsiannikov L.V., Group analysis of differential equations, Nauka, Moscow, 1978 (English transl.: Editor W.F. Ames, Academic Press, New York, 1982).

[8] Ibragimov N.H. (Editor), CRC Handbook of Lie group analysis of differential equations, Vols. 1, 2, 3, CRC Press, Boca Raton, 1994, 1995, 1996.

[9] Ovsyannikov L.V., The program "Submodels". Gas dynamics, Prikl. Mat. Mekh. 58 (1994), no. 4, 30-55 (English transl.: J. Appl. Math. Mech. 58 (1994), no. 4, 601-627).

[10] Meleshko S.V., Isentropic flows of an ideal gas, in Fragment, Vol. 401, Institute of Theoretical and Applied Mechanics, Institute of Hydrodynamics, Novosibirsk, 1989, 1-5.

[11] Hematulin A., Meleshko S.V., Gavrilyuk S.G., Group classification of one-dimensional equations of fluids with internal inertia, Math. Methods Appl. Sci. 30 (2007), 2101-2120.

[12] Ovsyannikov L.V., A singular vortex, Prikl. Mekh. Tekhn. Fiz. 36 (1995), no. 3, 45-52 (English transl.: J. Appl. Mech. Tech. Phys. 36 (1995), no. 3, 360-366). 
[13] Popovych H.V., On SO(3)-partially invariant solutions of the Euler equations, in Proceedings of Third International Conference "Symmetry in Nonlinear Mathematical Physics" (June 12-18, 1999, Kyiv), Editors A.G. Nikitin and V.M. Boyko, Proceedings of Institute of Mathematics, Kyiv 30 (2000), Part 1, $180-183$.

[14] Chupakhin A.P., Invariant submodels of a special vortex, Prikl. Mat. Mekh. 67 (2003), no. 3, 390-405 (English transl.: J. Appl. Math. Mech. 67 (2003), no. 3, 351-364).

[15] Cherevko A.A., Chupakhin A.P., A homogeneous singular vortex, Prikl. Mekh. Tekhn. Fiz. 45 (2004), no. 2, 75-89 (English transl.: J. Appl. Mech. Tech. Phys. 45 (2004), no. 2, 209-221).

[16] Pavlenko A.S., A projective submodel of the Ovsyannikov vortex, Prikl. Mekh. Tekhn. Fiz. 46 (2005), no. 4, 3-16 (English transl.: J. Appl. Mech. Tech. Phys. 46 (2005), no. 4, 459-470).

[17] Hematulin A., Meleshko S.V., Rotationally invariant and partially invariant flows of a viscous incompressible fluid and a viscous gas, Nonlinear Dynam. 28 (2002), 105-124.

[18] Golovin S.V., Invariant solutions of the singular vortex in magnetohydrodynamics, J. Phys. A: Math. Gen. 38 (2005), 8169-8184.

[19] Meleshko S.V., Methods for constructing exact solutions of partial differential equations, Springer, New York, 2005.

[20] Hearn A.C., REDUCE users manual, version 3.3, The Rand Corporation CP 78, Santa Monica, 1987.

[21] Bagderina Yu.Yu., Chupakhin A.P., Invariant and partially invariant solutions of the Green-Naghdi equations, Prikl. Mekh. Tekhn. Fiz. 46 (2005), no. 6, 26-35 (English transl.: J. Appl. Mech. Tech. Phys. 46 (2005), no. 6, 791-799).

[22] Ovsyannikov L.V., Optimal systems of subalgebras, Dokl. Akad. Nauk 333 (1993), no. 6, $702-704$ (English transl.: Russian Acad. Sci. Dokl. Math. 48 (1994), no. 3, 645-649). 